\title{
Squeezing in a $\Lambda$-type three-level atom via spontaneously generated coherence
}

\author{
Isabel Gonzalo, ${ }^{1}$ M. A. Antón, ${ }^{2}$ F. Carreño, ${ }^{2}$ and Oscar G. Calderón ${ }^{2,3}$ \\ ${ }^{1}$ Facultad de Ciencias Físicas, Universidad Complutense de Madrid, Ciudad Universitaria s/n, 28040, Spain \\ ${ }^{2}$ Escuela Universitaria de Óptica, Universidad Complutense de Madrid, C/ Arcos de Jalón s/n, 28037 Madrid, Spain \\ ${ }^{3}$ Stanford Picosecond FEL Center, Hansel Experimental Physics Laboratory, Stanford University, Stanford, \\ California 94305-4085, USA \\ (Received 15 July 2004; revised manuscript received 30 June 2005; published 13 September 2005)
}

\begin{abstract}
The squeezing spectrum of the fluorescent light is investigated for a laser-driven three-level atom of the $\Lambda$ configuration when quantum interference of the decay channels is accounted for. We show that when the two atomic transitions contribute to the detected fluorescence field, squeezing at certain frequency intervals is obtained in both the weak- and the high-Rabi-frequency regimes even for equally decay rates of the transitions. Unlike in two-level atoms in free space, squeezing can be obtained in both the in-phase and out-of-phase quadrature spectra although in different spectral regions. We also show that the squeezing spectrum can be controlled by an adequate selection of the Rabi frequencies and atomic detunings. Another remarkable effect is that squeezing can be achieved with proper relative phases of the driving fields. We provide an analytical description in the dressed basis which accounts for the main features of the squeezing spectra obtained from the numerical work.
\end{abstract}

DOI: 10.1103/PhysRevA.72.033809

PACS number(s): 42.50.Dv, 42.50.Gy, 42.50.Hz

\section{INTRODUCTION}

Resonance fluorescence is a central topic in quantum optics and has been shown to be a successful way to investigate the fundamentals of the interaction of matter and radiation [1-4]. Using this technique, a manifold of interesting phenomena have been observed or predicted such as photon antibunching [4-6], sub-Poissonian statistics [7], and squeezing [8], among others.

In contrast to fluorescence spectra which are detected without phase sensitivity, squeezing spectra are obtained by homodyne detection of scattered radiation from free atoms driven by a coherent field. In these experiments, the scattered radiation field of the atoms, $E$, is mixed with a local oscillator (LO) field $\left|E_{L O}\right| e^{i \theta}$, having a controllable fixed phase $\theta$, relative to the driving field. Thus, the signal $P(t)$ reaching the detector is proportional to the interference term between the LO and the scattered field, that is, $P(t) \propto\left|E_{L O}\right| x_{\theta}$, where $x_{\theta}$ is a quadrature of the scattered field given by $x_{\theta}=1 / 2\left(e^{-i \theta} E\right.$ $\left.+e^{i \theta} E^{\dagger}\right)$. Squeezing in the radiation field can be measured by analyzing the fluctuations in the detected power. In this way, the normally ordered variance of the quadrature components, either in total phase quadratures or in frequency components (squeezing spectrum), can be obtained. In the last case, the field must be frequency filtered $[9,10]$. Moreover, the phenomenon of phase-sensitive squeezing in resonance fluorescence, first predicted by Mandel [7] and Walls and Zöller [11], has received considerable attention in the context of two-level atoms. Theoretical calculations in a two-level atom have shown that the squeezing can be found in the out-ofphase quadrature component spectra in a weak coherent excitation regime under the condition $\Omega<2 \Gamma$ [12-14], where $\Omega$ and $\Gamma$ are the Rabi frequency and the atomic decay rate, respectively.

In spite of receiving considerable attention, squeezing in resonance fluorescence has eluded experimental observation, one of the problems being that atomic motion produces phase shifts which destroy the squeezing [15]. However, in a recent work [16], precision near-resonant phase-dependent spectra have been obtained by using a homodyne detection technique that suppresses excess noise by subtracting transmitted power signals from two identically prepared atomic samples. With this scheme, Lu et al. [16] and Zhao et al. [17] have found some evidence of squeezing when measuring the phase-dependent fluorescent spectra of coherently driven ${ }^{174} \mathrm{Yb}$ atoms at a phase near $\pm \pi / 4$ relative to the exciting field. This important result has renewed the exploration of phase-dependent spectra of resonance fluorescence in twolevel atoms. Since the fluorescence field quadrature components in two-level systems depend on the difference between the upper-level population and the square of the dipole moment [18], it was claimed [19-22] that three-level atoms of the $\Lambda$ type would be more suitable than two-level atoms, due to the possibility of reducing the upper-state population via the coherent population trapping effect. This dynamically induced atomic coherence also lead to many phenomena, such as electromagnetically induced transparency [23-25], lasing without inversion [26-28], refractive index enhancement without absorption [29-31], and giant nonlinearity [32-34]. Squeezing of the fluorescent field emitted by $\Lambda$-type atoms has been previously discussed in terms of the variance of quadrature components of the total electric field rather than in terms of the squeezing spectrum. Vogel and Blatt [15] found steady-state squeezing only when the two spontaneous emission rates and the two Rabi frequencies were significantly different from each other. Ficek et al. [22] also found that steady-state squeezing is limited to the case when the fluorescence of only one atomic transition is detected and the decay rate of the transition, which exclusively contributes to the detected fluorescence field, is larger than the decay of the other transition. Moreover, they did not find squeezing when the two atomic transitions equally contribute to the detected fluorescence field. 
In this paper we are interested in analyzing the effects of another way of generating coherence which connects with relaxation processes such as spontaneous emission. This spontaneously generated coherence (SGC) is responsible for many novel effects, such as narrow resonances and probe transparency [35-37], dark spectral lines [38,39], phasedependent line shapes [40,41], gain features, and dark transitions [42]. While spontaneously generated interference effects have been intensively studied in V-type atoms, such effects in $\Lambda$ systems have received considerably less attention. The existence of such spontaneously generated interference effects in $\Lambda$-type atoms, first predicted by Javananien [43], is not obvious due to the fact that there are two lower states as possible final states. For this reason, most of the work related to $\Lambda$ atoms did not include the effect of SGC. However, Hu and Peng [44] have recently demonstrated that a $\Lambda$ system with maximum quantum interference can be carried out using a dressed-state picture of a coherently driven $\mathrm{V}$ system without interference. It has been pointed out that SGC in a $\Lambda$-type atom can change the steady-state response of the medium $[43,45]$ and can significantly modify the absorption or spontaneous emission spectra of a neardegenerate system. Menon and Agarwal [46] have investigated the effects of SGC on a standard $\Lambda$ system and found that SGC brings about quantitative changes in line profiles when the two applied fields are both strong enough. Wu and Gao [47] have found that, in this system, the inversionless gain stems from both spontaneous generated coherence and dynamically induced coherence, although the former provides the major contribution to the inversionless gain. Joshi et al. [48] have demonstrated the controllability of atomic optical bistability in $\Lambda$ atoms by using SGC in the decay channels. Evers, Bullock, and Keitel [49] have discussed the effect of SGC on resonance fluorescence spectra in $\Lambda$-type atoms, when both transitions from the upper level to the lower levels are driven by a single laser field, and they found that the SGC term produces interesting effects such as the suppression of a dark state, which is present without interference, and the appearance of a narrow spectral feature in the fluorescence of the atom. Thus, it is expected that SGC may also greatly modify the squeezing spectrum.

In view of the experimental success in measuring the squeezing spectrum [16], in this paper we study the effect of spontaneous generated coherence on the squeezing spectrum of the fluorescent field. As far as we know, little research has been done in the phase-dependent spectra of three-level atoms, apart from the work of Gao et al. [50], which analyzes the effect of quantum interference on the squeezing spectrum in V-type atoms, and the work of Antón et al. [51] covering the squeezing spectrum in V-type atoms damped by a squeezed vacuum. We show that additional features in the squeezing spectrum emerge by the inclusion of SGC in a $\Lambda$-type system. With appropriate selection of the atomic parameters, we find that the squeezing spectrum is quite different from the case without SGC. In particular, we find that when a single coherent field couples both transitions, SGC causes squeezing otherwise not noticeable in absence of SGC. In the case of two coherent fields, each one coupled to a single transition, squeezing can be obtained for equal decay rates in the weak- and high-Rabi-frequency regimes in con- (a)

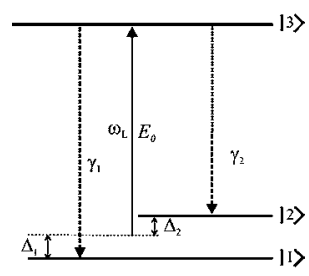

(b)

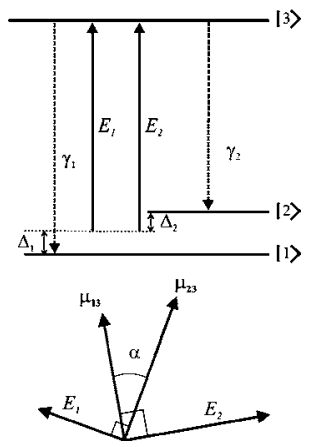

FIG. 1. A $\Lambda$-type atom driven by (a) a single-mode laser of frequency $\omega_{L}$ and (b) two coherent fields of the same frequency $\omega_{L}$. The polarization arrangement is also shown. $\gamma_{1}$ and $\gamma_{2}$ are the decay rates from the excited level to levels $|1\rangle$ and $|2\rangle$, respectively.

trast to the case of a two-level atom. The most remarkable effect is that the squeezing spectrum depends on the relative phase of the driving fields, so we can control the fluctuations by tuning the relative phase and/or the Rabi frequencies of the applied fields.

The paper is organized as follows. Section II establishes the model, i.e., the Hamiltonian of the system and the evolution equation of the atomic operators assuming the rotating-wave approximation and the squeezing spectrum is obtained in terms of the correlations of the density matrix. Numerical analysis showing the influence of SGC on the squeezing of the fluorescent field is presented in Sec. III. In Sec. IV, we develop an analysis in terms of the dressed states which aids in interpreting the numerical results. Finally, Sec. $\mathrm{V}$ summarizes the main conclusions.

\section{THE MODEL}

We consider a closed $\Lambda$-type three-level system with two near-degenerate levels $|1\rangle$ and $|2\rangle$, and one excited level $|3\rangle$ as shown in Fig. 1. The energies of the levels are $\hbar \omega_{i}(i$ $=1,2,3)$. Spontaneous and stimulated emissions between these states are governed by the interaction of the atom with a reservoir in a thermal field at zero temperature. In order to analyze the induced-coherence effects by spontaneous emission, the upper level $|3\rangle$ is coupled to the lower levels $|1\rangle$ and $|2\rangle$ by the same vacuum modes. The resonant frequencies between the upper level $|3\rangle$ and the ground levels $|2\rangle$ and $|1\rangle$ are $\omega_{32}$ and $\omega_{31}$, respectively. Note that $\omega_{31}-\omega_{32}=\omega_{21}$, is the frequency separation of the lower levels. Both transitions $|3\rangle \rightarrow|2\rangle$ and $|3\rangle \rightarrow|1\rangle$ are driven by an external coherent field $\vec{E}$ given by

$$
\vec{E}=\frac{1}{2} \vec{E}_{1}(t) e^{-i\left(\omega_{L} t+\phi_{1}\right)}+\frac{1}{2} \vec{E}_{2}(t) e^{-\left(i \omega_{L} t+\phi_{2}\right)}+\text { c.c. },
$$

where $\vec{E}_{j}(t)$ and $\phi_{j}(j=1,2)$ are the amplitudes and phases of the slowly varying field envelopes, respectively, and $\omega_{L}$ stands for the angular frequency of the fields.

The system is studied using the density matrix formalism. By following the traditional approach of Weisskopf and 
Wigner $[45,52,53]$, we have rederived the master equation for the reduced density matrix of the atomic system $\rho_{s}^{I}$, in the Born and Markov approximation and in the interaction picture

$$
\begin{aligned}
\frac{\partial \rho_{s}^{I}}{\partial t}= & -\frac{i}{\hbar}\left[H_{e x}^{I}, \rho_{s}^{I}\right]-\frac{1}{2} \gamma_{1}\left(\sigma_{33} \rho_{s}^{I}+\rho_{s}^{I} \sigma_{33}-2 \sigma_{13} \rho_{s}^{I} \sigma_{31}\right) \\
& -\frac{1}{2} \gamma_{2}\left(\sigma_{33} \rho_{s}^{I}+\rho_{s}^{I} \sigma_{33}-2 \sigma_{23} \rho_{s}^{I} \sigma_{32}\right) \\
& +\gamma_{12}\left(\sigma_{13} \rho_{s}^{I} \sigma_{32} e^{-i\left[\left(\omega_{31}-\omega_{32}\right) t+\delta\right]}+\sigma_{23} \rho_{s}^{I} \sigma_{31} e^{i\left[\left(\omega_{31}-\omega_{32}\right) t+\delta\right]}\right),
\end{aligned}
$$

where $H_{e x}^{I}$ is given by

$$
H_{e x}^{I}=\Delta_{1} \sigma_{33}+\left(\Delta_{1}-\Delta_{2}\right) \sigma_{22}-\hbar \sum_{m=1}^{2}\left[\Omega_{m} \sigma_{3 m}+\text { H.c. }\right] \text {. }
$$

The operators $\sigma_{m n}=|m\rangle\langle n|$ are the usual Pauli matrices, and $\vec{\mu}_{m 3}$ and $\Omega_{m}=\vec{\mu}_{m 3} \cdot \vec{E}_{m} / 2 \hbar(m=1,2)$ are the dipolar moment and the Rabi frequencies of the transitions $|m\rangle \rightarrow|3\rangle$, respectively. $\Delta_{1}=\omega_{31}-\omega_{L}$ and $\Delta_{2}=\omega_{32}-\omega_{L}$ are the detunings between the field and the optical transitions $|3\rangle \rightarrow|1\rangle$ and $|3\rangle$ $\rightarrow|2\rangle$, respectively; $\delta=\phi_{2}-\phi_{1}$ is the relative phase of the two coherent fields. The damping terms $\gamma_{1}$ and $\gamma_{2}$ are the decay rates for the $|3\rangle \rightarrow|1\rangle$ and $|3\rangle \rightarrow|2\rangle$ transitions, respectively. The damping terms proportional to $\gamma_{12}$ in Eq. (2.2) are particularly important when $\left(\omega_{31}-\omega_{32}\right) \simeq \gamma_{1}, \gamma_{2}$, and they arise due to the coupling of the two transitions $|3\rangle \rightarrow|1\rangle$ and $|3\rangle \rightarrow|2\rangle$ with the same vacuum mode. They are responsible for the quantum interference between the two decay channels. In addition, it can be shown [45] that

$$
\gamma_{12}=\sqrt{\gamma_{1} \gamma_{2}} p
$$

where $p \equiv\left(\vec{\mu}_{13} \cdot \vec{\mu}_{23}\right) /\left|\vec{\mu}_{13}\right|\left|\vec{\mu}_{23}\right|=\cos \alpha, \alpha$ being the angle between the dipole moments. Thus, the quantum interference is maximum if the transition moment $\vec{\mu}_{13}$ is parallel to $\vec{\mu}_{23}$, and it disappears if they are perpendicular. The ability to control $\gamma_{12}$ has been experimentally demonstrated [54] in sodium dimers by considering the superposition of singlet and triplet states due to spin-orbit coupling, although a conflicting result was obtained in [55]. Recently some novel methods to produce spontaneous coherence are discussed in the literature $[56,57]$.

The equations of motion of the elements of the density matrix in a rotating-wave frame read as

$$
\begin{aligned}
\frac{\partial \rho_{13}}{\partial t}= & -\left[\left(\gamma_{1}+\gamma_{2}\right) / 2-i \Delta_{1}\right] \rho_{13}+2 i \Omega_{1} \rho_{33}-i \Omega_{2} \rho_{12} \\
& +i \Omega_{1} \rho_{22}-i \Omega_{1}, \\
\frac{\partial \rho_{33}}{\partial t}=- & \left(\gamma_{1}+\gamma_{2}\right) \rho_{33}+i \Omega_{1}\left(\rho_{13}-\rho_{31}\right)+i \Omega_{2}\left(\rho_{23}-\rho_{32}\right),
\end{aligned}
$$

$$
\frac{\partial \rho_{12}}{\partial t}=+i \omega_{21} \rho_{12}-i \Omega_{2} \rho_{13}+p \sqrt{\gamma_{1} \gamma_{2}} e^{i \delta} \rho_{33}+i \Omega_{1} \rho_{32}
$$

$$
\begin{gathered}
\frac{\partial \rho_{22}}{\partial t}=\gamma_{2} \rho_{33}-i \Omega_{2}\left(\rho_{23}-\rho_{32}\right), \\
\frac{\partial \rho_{23}}{\partial t}=-\left[\left(\gamma_{1}+\gamma_{2}\right) / 2-i \Delta_{2}\right] \rho_{23}+i \Omega_{2} \rho_{33}-i \Omega_{1} \rho_{21}-i \Omega_{2} \rho_{22},
\end{gathered}
$$

where $\rho_{i j} \equiv\left\langle\sigma_{j i}\right\rangle$.

Based on the Bloch equations (2.5)-(2.9), we may calculate the optical properties of light scattered by the atom such as the squeezing spectrum. For this purpose, the operator of the electric field

$$
\vec{E}(\vec{r}, t)=\vec{E}^{+}(\vec{r}, t)+\vec{E}^{-}(\vec{r}, t),
$$

at the point $\vec{r}$ of the observation is required. It may be decomposed into free and source field parts, i.e.,

$$
\vec{E}^{+}(\vec{r}, t)=\vec{E}_{\text {free }}^{+}(\vec{r}, t)+\vec{E}_{s}^{+}(\vec{r}, t),
$$

$\vec{E}_{s}^{+}(\vec{r}, t)$ being the positive-frequency part of the fluorescent light emitted by the atom. In our case, for the $\Lambda$-type atom shown in Fig. 1, and in the radiation zone, the positivefrequency part of the fluorescent light emitted by the atom takes the form [58]

$$
\begin{aligned}
\vec{E}_{s}^{+}(\vec{r}, t)= & -\frac{1}{c^{2} r}\left[\omega_{31}^{2} \vec{n} \times\left(\vec{n} \times \vec{\mu}_{13}\right) \sigma_{13}\left(t^{\prime}\right)+\omega_{32}^{2} \vec{n}\right. \\
& \left.\times\left(\vec{n} \times \vec{\mu}_{23}\right) \sigma_{23}\left(t^{\prime}\right)\right] e^{-i \omega_{L} t^{\prime}},
\end{aligned}
$$

where $t^{\prime}=t-r / c$ is the retarded time, and $\vec{n}$ is a unit vector in the direction of observation. We will assume that $\omega_{31} \simeq \omega_{32}$ and $\vec{n}$ is perpendicular to the atomic dipole moments $\vec{\mu}_{13}$ and $\vec{\mu}_{23}$, so Eq. (2.12) can be rewritten as

$$
\vec{E}_{s}^{+}(\vec{r}, t)=f(r)\left[\vec{\mu}_{13} \sigma_{13}\left(t^{\prime}\right)+\vec{\mu}_{23} \sigma_{23}\left(t^{\prime}\right)\right] e^{-i \omega_{L} t^{\prime}}
$$

where $f(r)=\omega_{31}^{2} / c^{2} r$.

In squeezing measurements the normally ordered variance $\left\langle:\left(\Delta E_{\theta}\right)^{2}:\right\rangle$ is the magnitude of interest, $E_{\theta}$ being the slowly varying electric field operator modified due to the beating of the scattered field under study with a local oscillator with phase $\theta$,

$$
\begin{aligned}
\vec{E}_{\theta}(\vec{r}, t)= & \frac{1}{2} \vec{E}_{s}^{+}(\vec{r}, t) e^{i\left(\omega_{L} t+\theta\right)}+\frac{1}{2} \vec{E}_{s}^{-}(\vec{r}, t) e^{-i\left(\omega_{L} t+\theta\right)}=\vec{E}_{1}(\vec{r}, t) \cos \theta \\
& +\vec{E}_{2}(\vec{r}, t) \sin \theta
\end{aligned}
$$

where

$$
\vec{E}_{1}(\vec{r}, t)=\frac{1}{2} \vec{E}_{s}^{+}(\vec{r}, t) e^{i \omega_{L} t}+\frac{1}{2} \vec{E}_{s}^{-}(\vec{r}, t) e^{-i \omega_{L} t}
$$




$$
\vec{E}_{2}(\vec{r}, t)=\frac{i}{2} \vec{E}_{s}^{+}(\vec{r}, t) e^{i \omega_{L} t}-\frac{i}{2} \vec{E}_{s}^{-}(\vec{r}, t) e^{-i \omega_{L} t},
$$

are the in-phase and out-of-phase quadratures of the fluorescent field relative to the coherent driving field, respectively.

The normally and time-ordered variance of $E_{\theta}$ is defined as follows (see Ref. $[9,10,50]$ ):

$$
\begin{aligned}
\left\langle:\left(\Delta E_{\theta}(\vec{r}, t)\right)^{2}:\right\rangle= & \left(\frac{1}{2 \pi}\right)^{2} \int_{-\infty}^{\infty} d \omega_{1} d \omega_{2} \int_{-\infty}^{\infty} d t_{1} d t_{2} \\
& \times e^{-i\left[\omega_{1}\left(t-t_{1}\right)+\omega_{2}\left(t-t_{2}\right)\right]} T\left\langle: E_{\theta}\left(\vec{r}, t_{1}\right), E_{\theta}\left(\vec{r}, t_{2}\right):\right\rangle,
\end{aligned}
$$

where $\langle A, B\rangle=\langle A . B\rangle-\langle A\rangle\langle B\rangle$, and $T\langle::\rangle$ indicates the operator orderings which are further explained in Appendix A.

In the steady-state regime, Eq. (2.17) can be written as

$$
\begin{aligned}
\left\langle:\left(\Delta E_{\theta}(\vec{r}, t)\right)^{2}:\right\rangle= & \frac{1}{2 \pi} \int_{-\infty}^{\infty} d \omega \int_{\infty}^{-\infty} d \tau e^{-i \omega_{1} \tau} \\
& \times T\left\langle: E_{\theta}(\vec{r}, t), E_{\theta}(\vec{r}, t+\tau):\right\rangle .
\end{aligned}
$$

Since we are interested in the squeezing spectrum, following Knoll et al. [10] and Gao et al. [50], we introduce the squeezed spectral density

$$
\langle: S(\vec{r}, t, \theta):\rangle=\frac{1}{2 \pi} \int_{\infty}^{-\infty} d \tau e^{-i \omega_{1} \tau} T\left\langle: E_{\theta}(\vec{r}, t), E_{\theta}(\vec{r}, t+\tau):\right\rangle .
$$

Inserting the positive and negative parts of the fluorescent field of Eq. (2.13) into Eq. (2.19), we can express the spectrum as

$$
\begin{aligned}
\langle: S(\vec{r}, \omega, \theta):\rangle= & \frac{f(r)^{2}}{4 \pi} \operatorname{Re} \int_{0}^{\infty} d \tau\left(e^{i \omega \tau}+e^{-i \omega \tau}\right)\left\{\left[\mu _ { 1 3 } ^ { 2 } \left\langle\sigma_{13}(t\right.\right.\right. \\
& \left.+\tau), \sigma_{13}(t)\right\rangle+\vec{\mu}_{13} \cdot \vec{\mu}_{23}\left\langle\sigma_{13}(t+\tau), \sigma_{23}(t)\right\rangle \\
& +\vec{\mu}_{13} \cdot \vec{\mu}_{23}\left\langle\sigma_{23}(t+\tau), \sigma_{13}(t)\right\rangle+\mu_{23}^{2}\left\langle\sigma_{23}(t\right. \\
& \left.\left.+\tau), \sigma_{23}(t)\right\rangle\right] e^{i 2\left(\theta+\omega_{L} r / c\right)}+\mu_{13}^{2}\left\langle\sigma_{31}(t+\tau), \sigma_{13}(t)\right\rangle \\
& +\mu_{23}^{2}\left\langle\sigma_{32}(t+\tau), \sigma_{23}(t)\right\rangle+\vec{\mu}_{13} \cdot \vec{\mu}_{23}\left\langle\sigma_{31}(t\right. \\
& \left.\left.+\tau), \sigma_{23}(t)\right\rangle+\vec{\mu}_{13} \cdot \vec{\mu}_{23}\left\langle\sigma_{32}(t+\tau), \sigma_{13}(t)\right\rangle\right\} .
\end{aligned}
$$

The two-time correlation functions which appear in Eq. (2.20) are calculated in Appendix B where the final form of the spectrum is provided in a useful form for computational work.

\section{SQUEEZING SPECTRA OF THE FLUORESCENCE FIELD QUADRATURES: NUMERICAL RESULTS}

In this section we present numerical results concerning the effect of SGC on the behavior of the squeezing spectrum. In the calculations Rabi frequencies, detunings, and atomic splittings are in units of the spontaneous emission rate of the upper level $\left(\gamma_{1}=\gamma_{2} \equiv \gamma\right)$. We assume that $\left|\vec{\mu}_{13}\right|=\left|\vec{\mu}_{23}\right|$ for
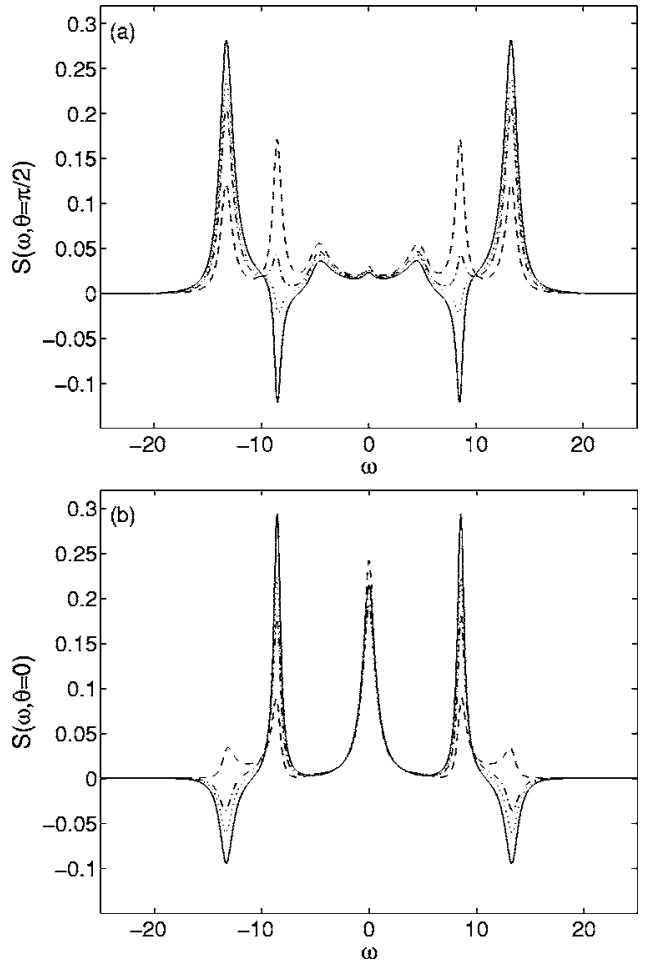

FIG. 2. Squeezing spectrum $S(\omega, \theta)$ as a function of $\omega$ for $\omega_{21}$ $=10 \gamma, \Delta_{2}=0$, and $\Omega=2.5 \gamma$. The curves correspond to $p=0$ (dashed line), 0.5 (dot-dashed line), 0.7 (dotted line), and 0.99 (solid line). $\theta=$ (a) $\pi / 2$ and (b) 0 .

simplicity. We also assume that $e^{2 i \omega_{L} r / c}=1$, and scale the squeezing spectrum by $\mu_{13}^{2} f^{2}(r) /(2 \pi \gamma)$. In the rest of the work $\omega$ stands for $\left(\omega-\omega_{L}\right) / \gamma$. We are interested in showing how different experimentally accessible parameters, such as Rabi frequencies or atomic detunings, can affect the squeezing spectrum. In the following analysis we compare the case without quantum interference $(p=0)$ with the case when quantum interference is present $(p \neq 0)$.

Let us consider the squeezing spectrum for the nondegenerate case $\omega_{21}=10 \gamma$, and $\Delta_{2}=\omega_{32}-\omega_{L}=0$, that is, the driving field is on resonance with the atomic transition $|3\rangle \rightarrow|2\rangle$. If a single field couples both transitions [see Fig. 1(a)] then $\Omega_{1}$ $=\Omega_{2} \equiv \Omega$.

We plot in Fig. 2(a) the out-of-phase quadrature spectrum $[S(\omega, \theta=\pi / 2)]$ versus the frequency $\omega$ when considering $\Omega$ $=2.5 \gamma$ and different degrees of quantum interference. The dashed, dot-dashed, dotted, and solid lines correspond to $p$ $=0,0.5,0.7$, and 0.99 , respectively. We call attention to the fact that the fluorescent field is squeezed in a selected quadrature $\theta$ and at a certain frequency $\omega$ if $S(\omega, \theta)<0$. In the absence of quantum interference (dashed line), the spectrum consists of seven peaks located at the driving laser frequency and the Rabi sidebands. The squeezing obtained is null or negligible (the minimum value is in the order of $\left.-10^{-4}\right)$. The spectral features are dramatically modified when considering the quantum interference between the decay channels. For $p$ larger than a certain threshold value $(p$ $>0.55$ ), two negative peaks appear at the high-frequency inner sidebands. Note that squeezing is obtained for Rabi 


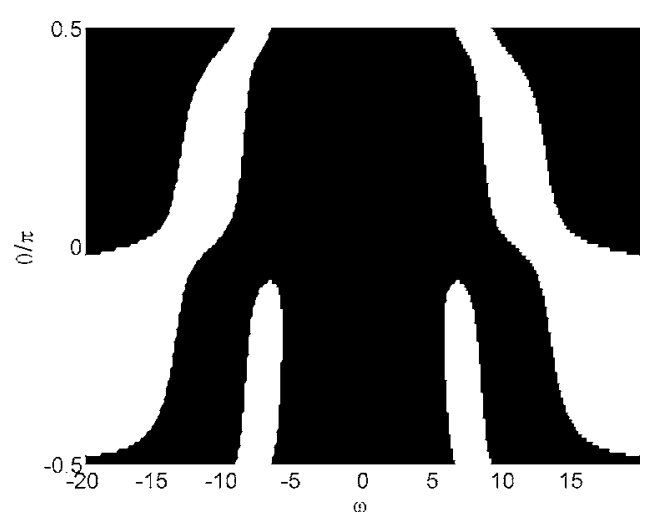

FIG. 3. State diagram in the plane $(\omega, \theta)$ showing the regions where squeezing appears (in white). The values of the squeezing vary within the interval $[-0.12,-0.001]$. Parameters used are $\omega_{21}$ $=10 \gamma, \Delta_{2}=0, \Omega=2.5 \gamma$, and $p=0.99$.

frequencies greater than $\Omega>2 \gamma$, in contrast to the two-level case where squeezing appears only for $\Omega<2 \gamma[12-14]$. For a very strong field, squeezing disappears for the out-of-phase quadrature spectrum as in two-level atoms. It is remarkable that squeezing appears in other quadratures as a consequence of SGC although at different Rabi sidebands. To illustrate this feature we plot in Fig. 2(b) the in-phase quadrature spectrum $[S(\omega, \theta=0)]$ versus the frequency $\omega$ when considering the rest of parameters as those used to produce Fig. 2(a). The numerical analysis of the in-phase quadrature spectrum reveals that there exists a different threshold value $(p>0.22)$ to obtain squeezing at the outer sideband. The new threshold value is lower than those found for the out-of-phase quadrature. Finally, the maximum level of squeezing at the in-phase (out-of-phase) quadrature is $-0.106(-0.089)$. In Fig. 3 we plot a state diagram in the $(\omega, \theta)$ plane showing the regions where squeezing is obtained. We observe that squeezing takes place in all quadratures. Furthermore we observe that the maximum reduction of fluctuations at different frequencies depends on the considered quadrature: when $\theta=0$ the squeezing is obtained at the outer sideband and by varying $\theta$ up to $\pi / 2$ the frequency at which the maximum squeezing is achieved changes from the outer sideband to the highfrequency inner sideband. In view of these results, we conclude that SGC plays a crucial role in the reduction of fluctuations beyond the standard limit and it opens the possibility for obtaining significant squeezing in the fluorescent signal. Similar results are obtained for other values of the splitting between the two lower levels. We have also checked the generality of our results by analyzing the squeezing spectra for different values of the detuning $\Delta_{2}$. We found that squeezing occurs for a wide range of parameters, not just the ones chosen in Figs. 2 and 3.

We now consider a scheme where two coherent fields are driving the atom. Note that $p<1$ due to the polarization arrangement in this configuration [see Fig. 1(b)]. In this case, we have more externally adjustable parameters to modify the squeezing spectrum than in the case of a single driving laser. Here we show that the detuning and the relative phase of the driving fields alter the squeezing spectrum almost at will.

First, we address the influence of the detuning $\Delta_{1}$ in the squeezing spectrum for $\Delta_{2}=0$. In Figs. 4(a) and 4(b) we
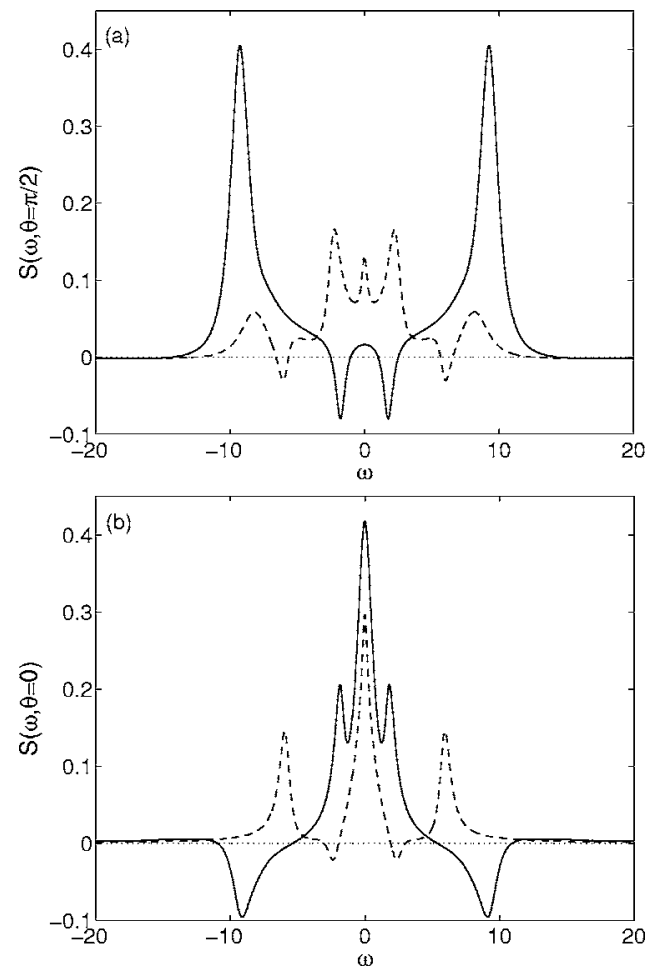

FIG. 4. Influence of the detuning $\Delta_{1}$ on the squeezing spectrum $S(\omega, \theta)$ as a function of $\omega$ for $\Delta_{2}=0, \Omega_{1}=1 \gamma, \Omega_{2}=4 \gamma, p=0.98$, and $\delta=0 . \Delta_{1}=0$ (dotted line), 2 (dashed line), and 5 (solid line). $\theta=$ (a) $\pi / 2$ and (b) 0 .

show the squeezing spectrum of the out-of-phase and inphase quadratures for different values of $\Delta_{1}$. In the case with $\Delta_{1}=0$ (dotted line) the fluorescence emission quenches completely and no squeezing is obtained since the system is in a trapping state. For $\Delta_{1} \neq 0$, the trapping state is destroyed and squeezing appears at the inner or outer sideband. Therefore, the frequency of the radiation at which squeezing occurs can be tuned by changing the atomic detuning, or the splitting of the two lower levels. We obtain again that squeezing appears in both quadratures as a consequence of SGC although at different frequencies. It should be remarked that in the absence of quantum interference no squeezing appears neither in the out-of-phase nor in the in-phase quadrature spectrum.

Now we resort to analyze how the relative phase of the driving fields influences the squeezing spectrum. In Figs. 5(a) and 5(b) the squeezing spectrum is shown for different values of the relative phase $\delta$ of the driving fields for the out-of-phase and in-phase quadratures. We assume a lower level splitting $\omega_{21}=5 \gamma, \Delta_{2}=0$, and Rabi frequencies $\Omega_{1}=\gamma$, and $\Omega_{2}=4 \gamma$. It is well known that, in the absence of SGC, the fluorescence spectrum of a $\Lambda$ atom does not depend on the relative phase between the two applied fields. However, when a system forms a closed loop, its dynamics becomes phase dependent $[40,41,59,60]$. We found that the squeezing spectrum is very sensitive to the relative phase $\delta$ of the driving fields. In the case presented in Fig. 5(a) squeezing appears in two peaks near the central region when $\delta=0$ (solid line) while for phase opposition $(\delta=\pi)$ no squeezing is obtained at any frequency (dashed line). For the case of the in-phase quadrature shown in Fig. 5(b) squeezing appears at 

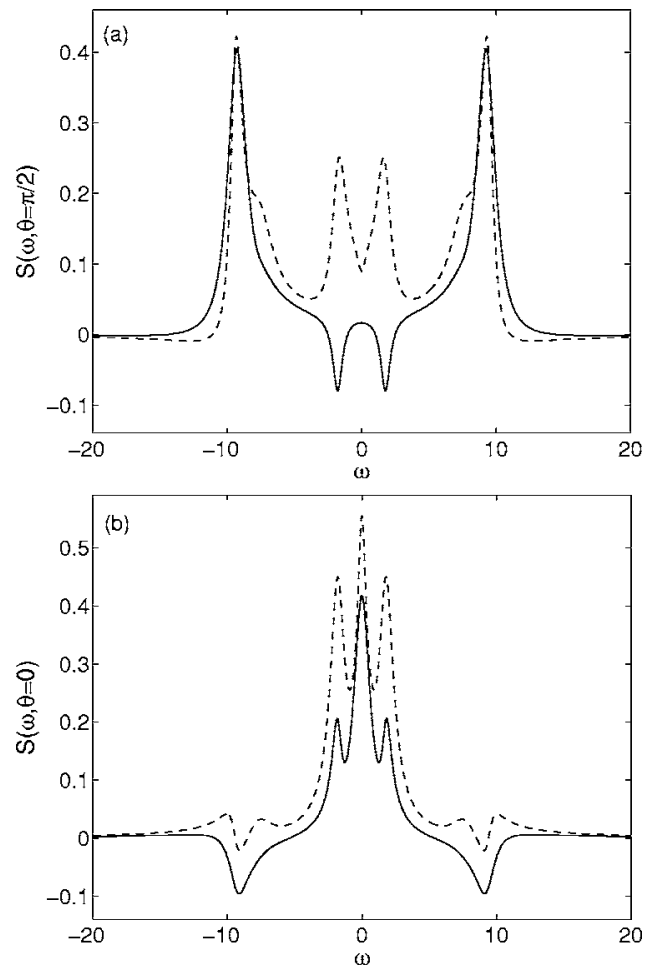

FIG. 5. Influence of the relative phase $\delta$ on the squeezing spectrum $S(\omega, \theta)$ as a function of $\omega$ for $\Delta_{2}=0, \Omega_{1}=1 \gamma, \Omega_{2}=4 \gamma, p$ $=0.98$, and $\omega_{21}=5 \gamma . \delta=0$ (solid line) and $\pi$ (dashed line). $\theta=(\mathrm{a})$ $\pi / 2$ and (b) 0 .

the outer sidebands. The main effect of changing the relative phase $\delta$ from 0 to $\pi$ when $\theta=0$ is to change the level of reduction of fluctuations at the outer sideband. We have checked that the strong reduction or even the disappearance of squeezing when the relative phase $\delta$ changes from 0 to $\pi$ is a general trends for all quadratures.

In summary, the presence of SGC allows the control of the squeezing features by modifying the detuning and/or the relative phase of the fields from zero to $\pi$. It is worth noting that squeezing can also be controlled by changing the strength of one of the driving fields, say for example $\Omega_{1}$, while maintaining fixed the other field $\left(\Omega_{2}\right)$.

\section{DRESSED-STATE EXPLANATION OF THE NUMERICAL RESULTS}

The objective of this section is to gain some insight in the numerical results presented in the previous section. In what follows we shall see that the squeezing is a direct consequence of the SGC. To this end we rewrite the squeezing spectrum (2.20) as the sum of two contributions, i.e., $\langle: S(\vec{r}, \omega, \theta):\rangle=S_{N I}(\omega)+S_{I}(\omega)$. The first and the second terms will be referred to as the noninterfering and interfering terms, respectively. We express these terms as

$$
\begin{gathered}
S_{N I}(\omega)=S_{1}(\omega)+S_{2}(\omega), \\
S_{I}(\omega)=p\left[S_{12}(\omega)+S_{21}(\omega)\right],
\end{gathered}
$$

where
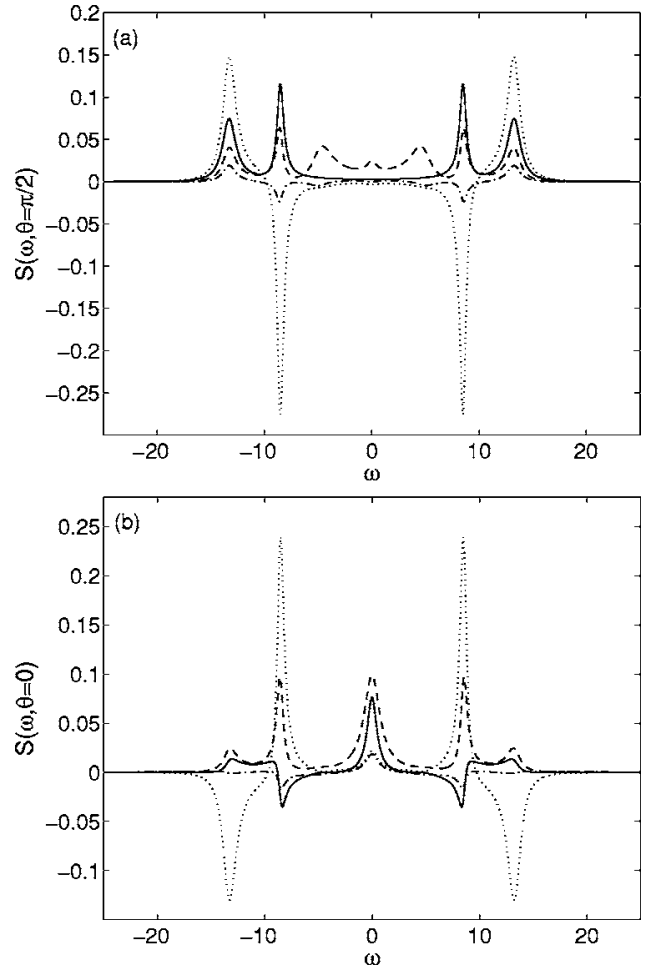

FIG. 6. Contributions of the different terms of the squeezing spectrum: $S_{1}(\omega)$ (solid line), $S_{2}(\omega)$ (dashed line), $S_{12}(\omega)$ (dashdotted line), and $S_{21}(\omega)$ (dotted line). In (a) [(b)] the data are those used to produce Fig. 2(a) [Fig. 2(b)] for the case $p=0.99$.

$$
\begin{aligned}
& S_{j}(\omega)= \frac{\left|\mu_{j 3}\right|^{2} f^{2}(r)}{4 \pi} \operatorname{Re} \int_{0}^{\infty} d \tau\left(e^{i \omega \tau}+e^{-i \omega \tau}\right) \\
& \times\left[\left\langle\sigma_{j 3}(t+\tau), \sigma_{j 3}(t)\right\rangle e^{i 2 \theta}+\left\langle\sigma_{3 j}(t+\tau), \sigma_{j 3}(t)\right\rangle\right], \\
& S_{j k}(\omega)= \frac{\left|\mu_{j 3}\right|\left|\mu_{k 3}\right| f^{2}(r)}{4 \pi} \operatorname{Re} \int_{0}^{\infty} d \tau\left(e^{i \omega \tau}+e^{-i \omega \tau}\right), \\
& \times\left[\left\langle\sigma_{j 3}(t+\tau), \sigma_{k 3}(t)\right\rangle e^{i 2 \theta}+\left\langle\sigma_{3 j}(t+\tau), \sigma_{k 3}(t)\right\rangle\right], \\
&(j, k=1,2) .
\end{aligned}
$$

Note that the explicit appearance of $p$ in $S_{I}(\omega)$ [see Eq. (4.2)] arises from the classical interference of the two fields radiated by the two dipoles. Furthermore, the quantum interference parameter $p$ is also incorporated in the correlations through the time evolution of density matrix elements. The noninterfering term involves correlations associated with single transitions $|3\rangle \rightarrow|1\rangle\left[S_{1}(\omega)\right]$ and $|3\rangle \rightarrow|2\rangle\left[S_{2}(\omega)\right]$, whereas the interfering term involves correlations of the two transitions. It is of interest to analyze how each term contributes to the squeezing spectrum. Thus, we plot in Figs. 6(a) and $6(\mathrm{~b})$ the different terms separately corresponding to the case in solid line in Figs. 2(a) and 2(b). We observe that the stronger fluctuations are associated with $|3\rangle \rightarrow|1\rangle$, thus we can approximate $S_{N I}(\omega) \approx S_{1}(\omega)$. On the other hand, the contributions to $S_{I}(\omega)$ are negative definite at the sidebands of interest, and the main contribution is associated with $S_{21}(\omega)$, 
then we can make the approximation $S_{I}(\omega) \approx p S_{21}(\omega)$. Therefore the squeezing at the high-frequency inner sideband (outer sideband) displayed in Figs. 2(a) and 2(b) is purely attributable to the quantum interference. In view of the previous considerations, the spectrum may be approximated by

$$
\langle: S(\vec{r}, \omega, \theta):\rangle \approx S_{1}(\omega)+p S_{21}(\omega) .
$$

To further explore the origin of the reduction of fluctuations we will work out the spectrum of Eq. (4.5) in the dressed-state basis, which are eigenstates of $H_{e x t}^{I}$ [see Eq. (2.3)]. While it is possible to treat the problem with full generality in the dressed-atom picture, in the interest of having transparent expressions to deal with, we restrict ourselves to the situations considered in previous section, where squeezing appears at the sidebands.

The eigenstates of $H_{e x t}^{I}$ [see Eq. (2.3)] can be written in terms of the bare states as

$$
\left(\begin{array}{l}
|\alpha\rangle \\
|\beta\rangle \\
|\gamma\rangle
\end{array}\right)=\left(\begin{array}{lll}
a_{1 \alpha} & a_{2 \alpha} & a_{3 \alpha} \\
a_{1 \beta} & a_{2 \beta} & a_{3 \beta} \\
a_{1 \gamma} & a_{2 \gamma} & a_{3 \gamma}
\end{array}\right)\left(\begin{array}{l}
|1\rangle \\
|2\rangle \\
|3\rangle
\end{array}\right),
$$

where the coefficients are explicitly given by

$$
\begin{aligned}
\left(\begin{array}{lll}
a_{1 \alpha} & a_{2 \alpha} & a_{3 \alpha} \\
a_{1 \beta} & a_{2 \beta} & a_{3 \beta} \\
a_{1 \gamma} & a_{2 \gamma} & a_{3 \gamma}
\end{array}\right) \\
\quad=\left(\begin{array}{lll}
\frac{\Omega_{1}\left(\lambda_{\alpha}-\Delta_{1}\right)}{D_{\alpha}} & \frac{\Omega_{2} \lambda_{\alpha}}{D_{\alpha}} & -\frac{\lambda_{\alpha}\left(\lambda_{\alpha}-\Delta_{1}\right)}{D_{\alpha}} \\
\frac{\Omega_{1}\left(\lambda_{\beta}-\Delta_{1}\right)}{D_{\beta}} & \frac{\Omega_{2} \lambda_{\beta}}{D_{\beta}}-\frac{\lambda_{\beta}\left(\lambda_{\beta}-\Delta_{1}\right)}{D_{\beta}} \\
\frac{\Omega_{1}\left(\lambda_{\gamma}-\Delta_{1}\right)}{D_{\gamma}} & \frac{\Omega_{2} \lambda_{\gamma}}{D_{\gamma}}-\frac{\lambda_{\gamma}\left(\lambda_{\gamma}-\Delta_{1}\right)}{D_{\gamma}}
\end{array}\right),
\end{aligned}
$$

with $D_{i}=\sqrt{\left(\lambda_{i}-\Delta_{1}\right)^{2}\left(\Omega_{1}^{2}+\lambda_{i}^{2}\right)+\left(\Omega_{2} \lambda_{i}\right)^{2}}(i=\alpha, \beta, \gamma)$. The eigenvalues $\lambda_{i}$ are the roots of the cubic equation

$$
\lambda^{3}-2 \Delta_{1} \lambda^{2}+\left[\Delta_{1}^{2}-\left(\Omega_{1}^{2}+\Omega_{2}^{2}\right)\right] \lambda+\Omega_{1}^{2} \Delta_{1}=0,
$$

and are given by $\lambda_{\alpha}>\lambda_{\beta}>\lambda_{\gamma}$. These eigenvalues give seven different frequencies of the fluorescence spectrum

$$
\omega_{i j}=\omega_{L}+\Omega_{i j},
$$

where $\Omega_{i j}=\lambda_{i}-\lambda_{j}(i, j=\alpha, \beta, \gamma)$. Specifically, for the case of Fig. 2, the eigenvalues obtained are $\lambda_{\alpha}=12.8 \gamma, \lambda_{\beta}=7.9 \gamma$, and $\lambda_{\gamma}=-0.6 \gamma$. These frequencies are in agreement with those of the spectral lines presented in Fig. 2: $\Omega_{\alpha \gamma}=13.4 \gamma, \Omega_{\beta \gamma}$ $=8.5 \gamma, \Omega_{\alpha \beta}=4.9 \gamma$. The corresponding energy diagram is shown in Fig. 7(a). In consequence, the squeezing found at the high-frequency inner sideband displayed in Fig. 2(a) arises from transitions $|\beta\rangle \rightarrow|\gamma\rangle$ and $|\gamma\rangle \rightarrow|\beta\rangle$, while that found at the outer sideband and displayed in Fig. 2(b) arises from transitions $|\alpha\rangle \rightarrow|\gamma\rangle$ and $|\gamma\rangle \rightarrow|\alpha\rangle$.

In order to obtain the intensities of the spectral lines and the condition for squeezing, we calculate populations and coherences of the dressed states. Note that the energy separation between the dressed states is not symmetrical with regard to state $|\beta\rangle$ [see Fig. 7(a)], thus when computing the (a)
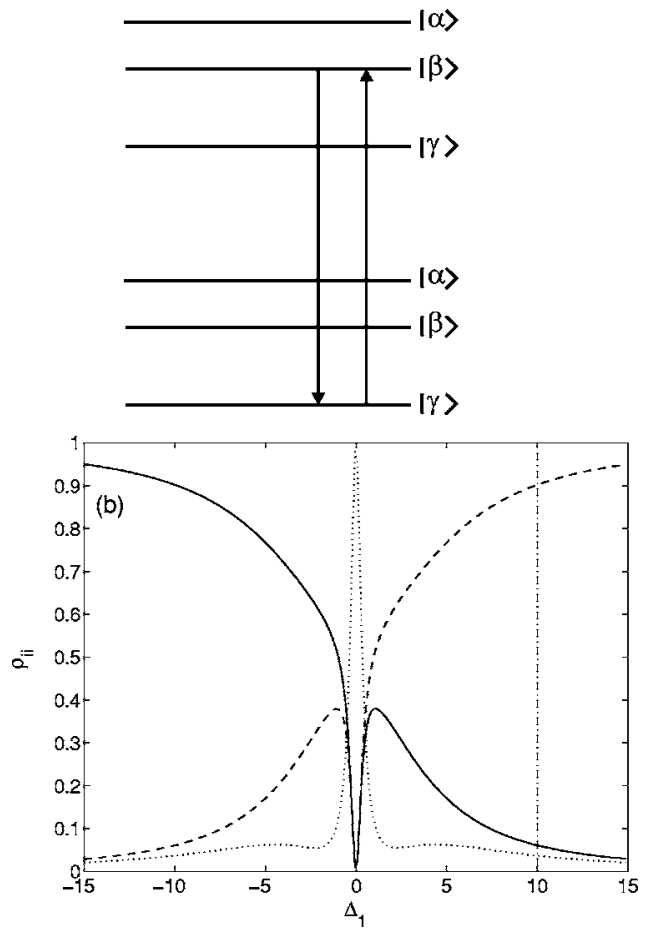

FIG. 7. (a) Relevant transitions between dressed states which account for the squeezing at the high-frequency inner sideband displayed in Fig. 2(a). (b) Populations of dressed states versus $\Delta_{1}$ for $\Delta_{2}=0$, and $\Omega_{1}=\Omega_{2}=2.5 \gamma$. Solid line, dotted line, and dashed line correspond to $\rho_{\alpha \alpha}, \rho_{\beta \beta}$, and $\rho_{\gamma \gamma}$, respectively. The vertical dashdotted line indicates the value of $\Delta_{1}$ used to produce Fig. 2.

time evolution of the coherences like $\rho_{i j}$ we only retain terms involving $\rho_{i j}(i, j=\alpha, \beta, \gamma)$. The equations of motion of the density matrix in the dressed-state picture and in the secular approximation read as

$$
\begin{aligned}
& \frac{d \rho_{\alpha \alpha}}{d t}=\Gamma_{0} \rho_{\alpha \alpha}+\Gamma_{1} \rho_{\gamma \gamma}+\Gamma_{2}, \\
& \frac{d \rho_{\gamma \gamma}}{d t}=\Gamma_{3} \rho_{\alpha \alpha}+\Gamma_{4} \rho_{\gamma \gamma}+\Gamma_{5}, \\
& \frac{d \rho_{\alpha \gamma}}{d t}=-\left(\Gamma_{\alpha \gamma}+i \Omega_{\alpha \gamma}\right) \rho_{\alpha \gamma}, \\
& \frac{d \rho_{\alpha \beta}}{d t}=-\left(\Gamma_{\alpha \beta}+i \Omega_{\alpha \beta}\right) \rho_{\alpha \beta}, \\
& \frac{d \rho_{\beta \gamma}}{d t}=-\left(\Gamma_{\beta \gamma}+i \Omega_{\beta \gamma}\right) \rho_{\beta \gamma},
\end{aligned}
$$

where the decay rates $\Gamma^{\prime}$ are given in Appendix C. Equation (4.10) reveals that the steady-state values of coherences are null. In addition, populations of the dressed states depend on the relative phase of the driving fields since the $\Gamma^{\prime}$ are shown to be phase dependent. In Fig. 7(b), we plot the dressed-state populations $\rho_{i i}(i=\alpha, \beta, \gamma)$ as a function of $\Delta_{1}$ for the case 
considered in the solid line of Fig. 2. It is seen that in the case $\Delta_{1}=0$, all the population is in the state $|\beta\rangle$. This is the population trapping effect dynamically induced by the coherent fields which leads to a complete quenching of the fluorescence emission. It is worth noting that in this situation, Menon and Agarwal [46] have shown that the SGC effect does not destroy the trapping state if the two fields have different amplitudes. For $\Delta_{1} \neq 0$, it can be concluded from Fig. 7(b) and Eq. (C1) that all dressed states are populated.

In the particular situation we are here addressing, the time evolution of the different coherences decouples each other in the high field limit, the problem of evaluating the squeezing spectrum becomes tractable. This simplification arises from the fact that steady-state correlations of the type $\left\langle\sigma_{i j}(0) \sigma_{k l}(0)\right\rangle$ are null for $i \neq l$ and $j \neq k$, where $i, j, k, l$ $=\alpha, \beta, \gamma$.
By inverting Eq. (4.6) we can easily obtain the atomic operators $\sigma_{i j}(\tau)$ in terms of the atomic operators in the dressed basis, i.e.,

$$
\begin{aligned}
\sigma_{i j}(\tau)= & a_{i \alpha} a_{j \alpha} \sigma_{\alpha \alpha}(\tau)+a_{i \beta} a_{j \beta} \sigma_{\beta \beta}(\tau)+a_{i \gamma} a_{j \gamma} \sigma_{\gamma \gamma}(\tau) \\
& +a_{i \alpha} a_{j \beta} \sigma_{\alpha \beta}(\tau)+a_{i \beta} a_{j \alpha} \sigma_{\beta \alpha}(\tau)+a_{i \alpha} a_{j \gamma} \sigma_{\alpha \gamma}(\tau) \\
& +a_{i \gamma} a_{j \alpha} \sigma_{\gamma \alpha}(\tau)+a_{i \beta} a_{j \gamma} \sigma_{\beta \gamma}(\tau)+a_{i \gamma} a_{j \beta} \sigma_{\gamma \beta}(\tau) \\
& (i, j=1,2,3) .
\end{aligned}
$$

The three first terms stand for the transitions that have the same frequency as that of the driving field, while the remaining terms describe the transitions between adjacent dressed states and they produce the different Rabi sidebands. By inserting Eq. (4.11) into Eq. (4.5), the squeezing spectrum of the out-of-phase and the in-phase quadratures reduce to

$$
\begin{aligned}
\left\langle: S\left(\vec{r}, \omega_{ \pm}, \pi / 2\right):\right\rangle= & \Gamma_{\beta \gamma} \frac{\left(a_{3 \beta} a_{1 \gamma}-a_{1 \beta} a_{3 \gamma}\right)\left[\left(a_{3 \beta} a_{1 \gamma}+p a_{3 \beta} a_{2 \gamma}\right) \rho_{\beta \beta}(0)-\left(a_{1 \beta} a_{3 \gamma}+p a_{2 \beta} a_{3 \gamma}\right) \rho_{\gamma \gamma}(0)\right]}{\Gamma_{\beta \gamma}^{2}+\left(\omega \mp \Omega_{\beta \gamma}\right)^{2}} \\
& +\Gamma_{\alpha \gamma} \frac{\left(a_{3 \alpha} a_{1 \gamma}-a_{1 \alpha} a_{3 \gamma}\right)\left[\left(a_{3 \alpha} a_{1 \gamma}+p a_{3 \alpha} a_{2 \gamma}\right) \rho_{\alpha \alpha}(0)-\left(a_{1 \alpha} a_{3 \gamma}+p a_{2 \alpha} a_{3 \gamma}\right) \rho_{\gamma \gamma}(0)\right]}{\Gamma_{\alpha \gamma}^{2}+\left(\omega \mp \Omega_{\alpha \gamma}\right)^{2}} \\
& +\Gamma_{\alpha \beta} \frac{\left(a_{3 \alpha} a_{1 \beta}-a_{1 \alpha} a_{3 \beta}\right)\left[\left(a_{3 \alpha} a_{1 \beta}+p a_{3 \alpha} a_{2 \beta}\right) \rho_{\alpha \alpha}(0)-\left(a_{1 \alpha} a_{3 \beta}+p a_{2 \alpha} a_{3 \beta}\right) \rho_{\beta \beta}(0)\right]}{\Gamma_{\alpha \beta}^{2}+\left(\omega \mp \Omega_{\alpha \beta}\right)^{2}}, \\
\left\langle: S\left(\vec{r}, \omega_{ \pm}, 0\right):\right\rangle= & \Gamma_{\beta \gamma} \frac{\left(a_{3 \beta} a_{1 \gamma}+a_{1 \beta} a_{3 \gamma}\right)\left[\left(a_{3 \beta} a_{1 \gamma}+p a_{3 \beta} a_{2 \gamma}\right) \rho_{\beta \beta}(0)+\left(a_{1 \beta} a_{3 \gamma}+p a_{2 \beta} a_{3 \gamma}\right) \rho_{\gamma \gamma}(0)\right]}{\Gamma_{\beta \gamma}^{2}+\left(\omega \mp \Omega_{\beta \gamma}\right)^{2}} \\
& +\Gamma_{\alpha \gamma} \frac{\left(a_{3 \alpha} a_{1 \gamma}+a_{1 \alpha} a_{3 \gamma}\right)\left[\left(a_{3 \alpha} a_{1 \gamma}+p a_{3 \alpha} a_{2 \gamma}\right) \rho_{\alpha \alpha}(0)+\left(a_{1 \alpha} a_{3 \gamma}+p a_{2 \alpha} a_{3 \gamma}\right) \rho_{\gamma \gamma}(0)\right]}{\Gamma_{\alpha \gamma}^{2}+\left(\omega \mp \Omega_{\alpha \gamma}\right)^{2}} \\
& +\Gamma_{\alpha \beta} \frac{\left(a_{3 \alpha} a_{1 \beta}+a_{1 \alpha} a_{3 \beta}\right)\left[\left(a_{3 \alpha} a_{1 b e t a}+p a_{3 \alpha} a_{2 \beta}\right) \rho_{\alpha \alpha}(0)+\left(a_{1 \alpha} a_{3 \beta}+p a_{2 \alpha} a_{3 \beta}\right) \rho_{\beta \beta}(0)\right]}{\Gamma_{\alpha \beta}^{2}+\left(\omega \mp \Omega_{\alpha \beta}\right)^{2}}+\frac{A_{\alpha \beta \gamma}}{\Gamma_{\alpha \beta \gamma}^{2}+\omega^{2}},
\end{aligned}
$$

where the subindex $+(-)$ stands for the positive $(\omega>0)$ [negative $(\omega<0)]$ part of the spectrum. Explicit expressions for $\Gamma_{\alpha \beta \gamma}$ and $A_{\alpha \beta \gamma}$ in Eq. (4.13) can be derived in a straightforward manner, although we do not provide them since they are related to a Lorentzian centered at the laser frequency where no squeezing is obtained for any quadrature.

From Eqs. (4.12) and (4.13) it can be concluded that the condition for obtaining squeezing at a certain sideband is that the numerator of the corresponding Lorentzian must be negative. Note that the numerators of the Lorentzians in Eqs. (4.12) and (4.13) depend explicitly on $p$ and on the steadystate populations of dressed states.

By using Eq. (4.12) and the expressions for the coefficients $a_{j k}(j=1,2,3),(k=\alpha, \beta, \gamma)$ given in Eq. (4.7), we can explain how squeezing originates in the situation displayed with the solid line in Fig. 2(a). In this particular case the squeezing occurs due to transitions $|\beta\rangle \rightarrow|\gamma\rangle$ and $|\gamma\rangle \rightarrow|\beta\rangle$. This means that we must only retain the first term of Eq. (4.12) which corresponds to the required transitions. In the case of Fig. 2(a), where $\Delta_{1}>\Omega_{R}$, with $\Omega_{R}=\sqrt{\Omega_{1}^{2}+\Omega_{2}^{2}}$, the eigenvalues obtained from Eq. (4.8) can be estimated as $\lambda_{\alpha}$ $\simeq \Delta_{1}+\Omega_{R}, \lambda_{\beta} \simeq \Delta_{1}-\Omega_{R}$, and $\lambda_{\gamma} \simeq-\Omega_{1}^{2} \Delta_{1} /\left(\Delta_{1}^{2}-\Omega_{R}^{2}\right)$. Using these eigenvalues, the squeezing spectrum of the out-ofphase quadrature at the high-frequency inner sideband can be roughly written as

$$
\begin{aligned}
\langle: S(\vec{r}, \omega= & \left.\left.\mp \Omega_{\beta \gamma}, \pi / 2\right):\right\rangle \simeq \frac{\Gamma_{\beta \gamma}}{\Gamma_{\beta \gamma}^{2}+\left(\omega \mp \Omega_{\beta \gamma}\right)^{2}}\left\{\Omega_{R}\left(\Delta_{1}-\Omega_{R}\right)\right. \\
& \times\left(\Delta_{1}^{2}-\Omega_{R}^{2}+p \Omega_{1} \Omega_{2}\right) \rho_{\beta \beta}(0) \\
& \left.-\Delta_{1} \Omega_{1}\left[p \Omega_{2}\left(\Delta_{1}-\Omega_{R}\right)-\Omega_{1} \Omega_{R}\right] \rho_{\gamma \gamma}(0)\right\} .
\end{aligned}
$$

Some consequences can be derived from Eq. (4.14). First of all, it is obvious that in the absence of quantum interference $(p=0)$, the squeezing spectrum is positive and no squeezing is possible. This explains the results obtained in Fig. 2(a) (dashed line), and it clearly points out that quantum interfer- 
ence is a necessary condition for obtaining squeezing. Moreover, the strength of quantum interference needed to produce squeezing must be greater than a certain value. Note that Eq. (4.14) consists of two terms: the first one is proportional to the population of the dressed state $|\beta\rangle$ and is always positive, whereas the second term is proportional to the population of the lower level $|\gamma\rangle$ and its sign can be positive or negative depending on the strength of the quantum interference. So in order to obtain squeezing, two different conditions must be satisfied: (1) the second term must be negative and (2) its absolute value must be larger than that of the first term. The first condition gives us a threshold value for the strength of the quantum interference

$$
p>\frac{\Omega_{1} \Omega_{R}}{\Omega_{2}\left(\Delta_{1}-\Omega_{R}\right)},
$$

which is easily reachable with a moderate quantum interference strength. As example, for the case of solid line in Fig. 2(a), Eq. (4.15) provides a threshold value of $p$ approximately equal to 0.55 , which agrees with the numerical calculations shown in Fig. 2(a). The second condition to obtain squeezing can be physically interpreted as follows: the population of the lower dressed level must be larger than in the second dressed level, i.e., $\rho_{\gamma \gamma}(0) \gg \rho_{\beta \beta}(0)$. This condition indicates that fluctuations are reduced beyond the standard limit when most of the population is in the dressed ground level and therefore spontaneous emission is significantly diminished. By looking again at Fig. 7(b), we see that, effectively, the lower level is highly populated at large detunings, which demonstrates the appearance of squeezing. However, this situation changes as we approach to the resonant condition, i.e., as $\Delta_{1}$ approaches to zero. This means that squeezing is not expected at detunings close to zero, in agreement with the numerical findings.

Now let us consider the results obtained for the in-phase quadrature as displayed in Fig. 2(b). In this case the squeezing is obtained at the outer sidebands which originate from transitions $|\alpha\rangle \rightarrow|\gamma\rangle$ and $|\gamma\rangle \rightarrow|\alpha\rangle$; thus we must retain the second term of Eq. (4.13). In this case the squeezing spectrum reduces to

$$
\begin{aligned}
\langle: S(\vec{r}, \omega= & \left.\left.\mp \Omega_{\alpha \gamma}, 0\right):\right\rangle \simeq \frac{\Gamma_{\alpha \gamma}}{\Gamma_{\alpha \gamma}^{2}+\left[\omega \mp \Omega_{\alpha \gamma}\right]^{2}}\left[\Omega_{R} \Delta_{1}^{2} \rho_{\alpha \alpha}(0)\right. \\
& \left.-\Omega_{1}\left(\Omega_{1} \Omega_{R}+p \Omega_{2} \Delta_{1}\right) \rho_{\gamma \gamma}(0)\right] .
\end{aligned}
$$

To obtain squeezing at the outer sideband it is necessary that the numerator in Eq. (4.16) should be negative. Thus the following approximate inequality must be satisfied for the case of a single driving laser $\left(\Omega_{1}=\Omega_{2} \equiv \Omega\right)$ :

$$
\rho_{\alpha \alpha}(0) \leqslant \frac{p \Omega}{\Delta_{1}} \rho_{\gamma \gamma}(0)
$$

In this case the fluctuations are reduced beyond the standard limit when most of the population is accumulated in the lower dressed level $|\gamma\rangle$ in comparison with that in level $|\alpha\rangle$. This is in accordance with the numerical results presented in Figs. 7(b) and 2(b).

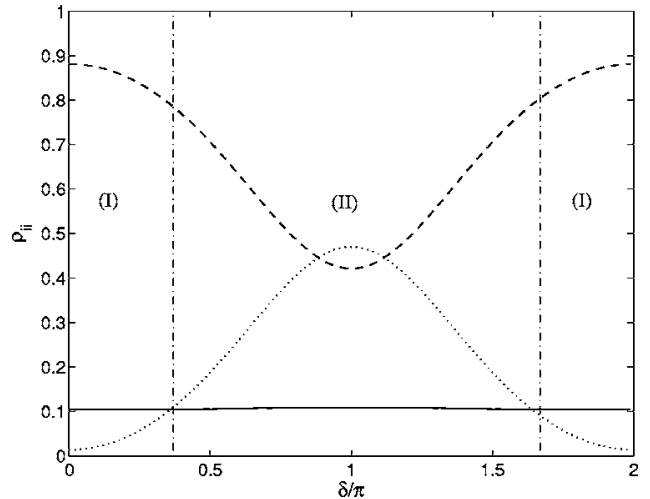

FIG. 8. Populations of dressed states versus $\delta$ for $\Delta_{2}=0, \Omega_{1}$ $=\gamma, \Omega_{2}=4 \gamma$, and $\omega_{21}=5 \gamma$. Solid line, dotted line, and dashed line correspond to $\rho_{\alpha \alpha}, \rho_{\beta \beta}$, and $\rho_{\gamma \gamma}$, respectively. The vertical dashdotted lines establish two regions for $\delta$, regions I and II, where squeezing is or is not obtained for the out-of-phase quadrature, respectively.

Finally, the dependence of the squeezing spectrum on the relative phase of the driving fields $(\delta)$ shown in Fig. 5 can be understood by considering the behavior of dressed-state populations. To this end we plot in Fig. 8 the corresponding populations as a function of $\delta$. From the squeezing spectrum given by Eqs. (4.14) and (4.16) we can see that squeezing appears when the respective numerators take negative values. These conditions strongly depend on the steady-state relative populations of states $|\gamma\rangle$ and $|\beta\rangle$ for the out-of phase quadrature and those of states $|\gamma\rangle$ and $|\alpha\rangle$ for the in-phase quadrature, provided $p$ is greater than a certain threshold value, so we can conclude that the dependence of the squeezing spectrum on $\delta$ is fully attributable to the dependence of populations on $\delta$ (see Appendix C). From Fig. 8 it can be appreciated that the population of the level $|\alpha\rangle$ does not change with $\delta$, its value being close to 0.1 , whereas the other two populations depend on $\delta$. When the two external fields are in phase $(\delta=0)$, the bulk of the population is concentrated in state $|\gamma\rangle$, and there is little population in state $|\beta\rangle$, taking negative values in the numerators of Eqs. (4.14) and (4.16). This explain the appearance of squeezing at the highfrequency inner (outer) sideband (see Fig. 5) for the out-ofphase (in-phase) quadrature. By increasing $\delta$ from 0 to $\pi$, population tends to accumulate in state $|\beta\rangle$ with the simultaneous reduction of population in state $|\gamma\rangle$ which reduces squeezing in both quadratures, in agreement with the numerical results shown in Fig. 5(a). In fact, when $\delta$ is larger than a threshold value $\delta>0.35 \pi$, squeezing disappears in the outof-phase quadrature, because the population $\rho_{\gamma \gamma}$ approximate to $\rho_{\beta \beta}$, in agreement with Fig. 5(a). This value of $\delta$ corresponds to a situation at which $\rho_{\gamma \gamma}(0) \approx 3.5 \rho_{\beta \beta}(0)$. Therefore, to obtain squeezing at the high-frequency inner sideband, population of dressed lower level must be more than three times larger than that of the intermediate level. However the value of the population in the dressed state $|\gamma\rangle$ when $\delta$ changes still satisfies condition (4.17), so the fluorescent field exhibits squeezing at the outer sideband for any value of $\delta$, i.e., squeezing is obtained in regions I and II for the in-phase quadrature, in accordance with the numerical results presented in Fig. 5(b). 
One may wonder if the disappearance of the squeezing at a certain frequency when changing the relative phase of the driving fields is accompanied by the generation of squeezing at other quadratures at the same frequency. In order to analyze this question we have carried out a numerical analysis for the case of the low-frequency inner sideband displayed as a solid line in Fig. 5(a) found at $\omega=1.8 \gamma$. Note that according to the previous discussion when $\delta>0.35 \pi$ there is no squeezing when considering $\theta=\pi / 2$ since we have changed from region I to region II in Fig. 8. We have computed the value of $S(\omega=1.8 \gamma, \theta)$ for several values of $\theta$ ranging from $\pi / 2$ to 0 by considering values of $\delta$ in regions I and II close to $\delta=0.35 \pi$, and no squeezing was found. We can conclude that the disappearance of squeezing at a certain frequency when varying $\delta$ is not accompanied by the generation of squeezing at other quadratures when considering the same frequency.

In summary, we can conclude that the squeezing characteristics can be modified by changing the dressed population distribution, which in turn can be achieved by changing Rabi frequencies, optical detunings or the relative phase of the driving fields.

\section{CONCLUSIONS}

In this work we present a theoretical investigation of the interaction of a three-level $\Lambda$-type atom with a coherent field when SGC is accounted for. The analysis is carried out using standard density matrix techniques that describe the evolution of atomic variables and incorporates the coupling with the vacuum at zero temperature. A key component of the system is the assumption that both lower levels are coupled to the same modes of the vacuum field. This assumption gives rise to the occurrence of well-known quantum interference in the atomic decay, the so-called SGC. The effects of SGC on the squeezing phase-dependent resonance fluorescence are analyzed in detail. We find that significant squeezing can be obtained in contrast to the usual case where interference between the two decay channels is not included in the atomic model.

When the atom is driven by a single field, we find that SGC allows us to obtain squeezing at the Rabi sidebands in the out-of-phase and in-phase quadratures. Furthermore, squeezing can be obtained in the high-Rabi-frequency regime. This result is of interest from an experimental point of view, since it should allow the observation of the reduction of fluctuations under nonrestrictive experimental conditions. We also show that the appearance of squeezing in the fluorescent field depends on the value of the quantum interference parameter $p$. There is a threshold value of $p$ which makes possible the obtention of squeezing. Finally, it should be remarked that squeezing is obtained when considering that the decay rates are identical, in contrast to other situations previously analyzed by Vogel and Blatt [15] and by Ficek et al. [22], where very different decay rates of the atomic transitions were considered.

When the atom is driven by two fields, and each field couples one transition, squeezing can be obtained in a wide spectral region by changing the Rabi frequencies of the fields. We also show that the phase-dependent resonance fluorescence spectrum is very sensitive to the relative phase of the two fields. The level of squeezing can be controlled by tuning the relative phase from zero to $\pi$.

Finally, we have analyzed the squeezing spectrum in the dressed-state basis. We have obtained analytical expressions which allow us to interpret the numerical results in terms of the relative populations of the dressed states. We show that squeezing takes place when the bulk of the population is concentrated in the dressed ground level and hence spontaneous emission diminishes appropriately.

In summary, we have shown the controllability of the squeezing spectrum by changing the SGC parameter, the amplitude of the Rabi frequencies, the detunings, or the relative phase of the fields.

\section{ACKNOWLEDGMENTS}

We are gratefully to Tomás Lorca L. Aranguren for a careful reading of the manuscript. This work was supported by the Projects No. PR3/04-12458 (UCM, Spain) and No. FIS2004-03267 (MEC, Spain).

\section{APPENDIX A: NORMAL-ORDERING AND TIME- ORDERING CRITERIA}

The normal and time orderings indicated by $T\langle::\rangle$ in Eq. (2.17) are necessary to ensure that each correlation function of the positive- and negative-frequency parts of the electric field in $\left\langle:\left[\Delta E_{\theta}(\vec{r}, t)\right]^{2}:\right\rangle$ has a correct time order and is measurable $[9,10]$. Note that in Eq. (2.17) the normal ordering is closely related to the basic mechanism of light absorption in ordinary photodetection, and time ordering reflects the fact that the time-dependent field commutation relations differ from the corresponding free-field commutation relations in the so-called time-delayed terms, which in general do not vanish [see Eq. (2.191) in [10]]. The reason to carry out the mentioned operations relies in the fact that source fields and free fields do not commute, in general, at different times. Time ordering allows us to express the correlations in terms of the source fields only, provided that correlations of the type $\left\langle\cdots E_{\text {free }}^{+}\right\rangle,\left\langle E_{\text {free }}^{-} \cdots\right\rangle$ are zero at the detector. Thus $T\langle::\rangle$ in Eq. (2.17) indicates the following operator orderings.

(1) There is normal ordering of the operators $E^{+}(r, t)$ and $E^{-}(r, t)$, with the operators $E^{-}(r, t)$ to the left of the operators $E^{+}(r, t)$.

(2) Time-ordering operator over a given set of normally ordered correlations acts as follows:

$$
\begin{aligned}
& T\left[E_{1}^{-}\left(t_{1}\right) E_{2}^{-}\left(t_{2}\right) \cdots E_{n}^{-}\left(t_{n}\right) E_{n+1}^{+}\left(t_{n+1}\right) E_{n+2}^{+}\left(t_{n+2}\right) \cdots E_{n+m}^{+}\left(t_{n+m}\right)\right] \\
&= T_{-}\left[E_{1}^{-}\left(t_{1}\right) E_{2}^{-}\left(t_{2}\right) \cdots E_{n}^{-}\left(t_{n}\right)\right] \\
& \times T_{+}\left[E_{n+1}^{+}\left(t_{n+1}\right) E_{n+2}^{+}\left(t_{n+2}\right) \cdots E_{n+m}^{+}\left(t_{n+m}\right)\right],
\end{aligned}
$$

where $T_{ \pm}$are defined as follows [10]:

$$
T_{-}\left[E_{1}^{-}\left(t_{1}\right) E_{2}^{-}\left(t_{2}\right) \cdots E_{n}^{-}\left(t_{n}\right)\right]=E_{i_{1}}^{-}\left(t_{i_{1}}\right) E_{i_{2}}^{-}\left(t_{i_{2}}\right) \cdots E_{i_{n}}^{-}\left(t_{i_{n}}\right),
$$




$$
\begin{gathered}
t_{i_{1}}<t_{i_{2}}<\cdots<t_{i_{n}}, \\
T_{+}\left[E_{n+1}^{+}\left(t_{n+1}\right) E_{n+2}^{+}\left(t_{n+2}\right) \cdots E_{n+m}^{+}\left(t_{n+m}\right)\right] \\
=E_{i_{n+1}}^{+}\left(t_{i_{n+1}}\right) E_{i_{n+2}^{+}}^{+}\left(t_{i_{n+2}}\right) \cdots E_{i_{n+m}^{+}}^{+}\left(t_{i_{n+m}}\right), \\
t_{i_{n+1}}>t_{i_{n+2}}>\cdots>t_{i_{n+m}} .
\end{gathered}
$$

Thus, taking into account the defined action of the timeordering operator $T$, the integrand of Eq. (2.17) takes the form

$$
\begin{aligned}
& T\left\langle: E_{\theta}\left(\vec{r}, t_{1}\right), E_{\theta}\left(\vec{r}, t_{2}\right):\right\rangle \\
&=\frac{1}{4}\left\{\left\langle\vec{E}_{s}^{+}\left(\vec{r}, t_{1}\right), \vec{E}_{s}^{+}\left(\vec{r}, t_{2}\right)\right\rangle e^{i\left[\omega_{L}\left(t_{1}+t_{2}\right)+2 \theta\right]} \Theta\left(t_{1}-t_{2}\right)\right. \\
&+\left\langle\vec{E}_{s}^{+}\left(\vec{r}, t_{2}\right), \vec{E}_{s}^{+}\left(\vec{r}, t_{1}\right)\right\rangle e^{i\left[\omega_{L}\left(t_{1}+t_{2}\right)+2 \theta\right]} \Theta\left(t_{2}-t_{1}\right) \\
&+\left\langle\vec{E}_{s}^{-}\left(\vec{r}, t_{1}\right), \vec{E}_{s}^{-}\left(\vec{r}, t_{2}\right)\right\rangle e^{-i\left[\omega_{L}\left(t_{1}+t_{2}\right)+2 \theta\right]} \Theta\left(t_{1}-t_{2}\right) \\
&+\left\langle\vec{E}_{s}^{-}\left(\vec{r}, t_{2}\right), \vec{E}_{s}^{-}\left(\vec{r}, t_{1}\right)\right\rangle e^{-i\left[\omega_{L}\left(t_{1}+t_{2}\right)+2 \theta\right]} \Theta\left(t_{2}-t_{1}\right) \\
&+\left\langle\vec{E}_{s}^{-}\left(\vec{r}, t_{1}\right), \vec{E}_{s}^{+}\left(\vec{r}, t_{2}\right)\right\rangle e^{i \omega_{L}\left(t_{2}-t_{1}\right)}\left[\Theta\left(t_{1}-t_{2}\right)+\Theta\left(t_{2}-t_{1}\right]\right) \\
&\left.+\left\langle\vec{E}_{s}^{-}\left(\vec{r}, t_{2}\right), \vec{E}_{s}^{+}\left(\vec{r}, t_{1}\right)\right\rangle e^{i \omega_{L}\left(t_{1}-t_{2}\right)}\left[\Theta\left(t_{1}-t_{2}\right)+\Theta\left(t_{2}-t_{1}\right)\right]\right\} .
\end{aligned}
$$

\section{APPENDIX B: STEADY-STATE SQUEEZING SPECTRUM}

The set of equations (2.5)-(2.9) can be written in matrix form as

$$
\frac{d \vec{\rho}}{d t}=B \vec{\rho}+\vec{C}
$$

with $\vec{\rho}$ defined as

$$
\begin{aligned}
\vec{\rho}= & {\left[\left\langle\sigma_{31}(t)\right\rangle,\left\langle\sigma_{13}(t)\right\rangle,\left\langle\sigma_{33}(t)\right\rangle,\left\langle\sigma_{21}(t)\right\rangle,\left\langle\sigma_{12}(t)\right\rangle,\right.} \\
& \left.\times\left\langle\sigma_{22}(t)\right\rangle,\left\langle\sigma_{32}(t)\right\rangle,\left\langle\sigma_{23}(t)\right\rangle\right]^{T},
\end{aligned}
$$

where $\left\langle\sigma_{k l}(t)\right\rangle=\rho_{l k}$, and the superscript $T$ stands for the transpose. The $B$ matrix is an $8 \times 8$ time-independent evolution matrix easily obtained from the coefficients Eqs. (2.5)-(2.9).

In order to obtain the two-time correlation functions in Eq. (2.20), we introduce the deviation $\Delta \sigma_{i j}$ of the dipole polarization operator from its mean steady-state value

$$
\Delta \sigma_{i j}\left(t^{\prime}\right)=\sigma_{i j}\left(t^{\prime}\right)-\left\langle\sigma_{i j}(\infty)\right\rangle, \quad i, j=2,3,
$$

which obviously satisfies

$$
\frac{d\left\langle\Delta \sigma_{i j}(\tau)\right\rangle}{d \tau}=B\left\langle\Delta \sigma_{i j}(\tau)\right\rangle, \quad i, j=1,2,3
$$

The two-time correlation function of the deviations can be obtained by invoking the quantum regression theorem [52] together with the optical Bloch equations (2.5)-(2.9). To do this, it is practical to define the column vectors

$$
\begin{aligned}
& \hat{U}^{(i j)}(\tau)= {\left[\left\langle\Delta \sigma_{31}(\tau) \Delta \sigma_{i j}(0)\right\rangle,\left\langle\Delta \sigma_{13}(\tau) \Delta \sigma_{i j}(0)\right\rangle,\right.} \\
& \times\left\langle\Delta \sigma_{33}(\tau) \Delta \sigma_{i j}(0)\right\rangle,\left\langle\Delta \sigma_{21}(\tau) \Delta \sigma_{i j}(0)\right\rangle, \\
& \times\left\langle\Delta \sigma_{12}(\tau) \Delta \sigma_{i j}(0)\right\rangle,\left\langle\Delta \sigma_{22}(\tau) \Delta \sigma_{i j}(0)\right\rangle, \\
&\left.\times\left\langle\Delta \sigma_{32}(\tau) \Delta \sigma_{i j}(0)\right\rangle,\left\langle\Delta \sigma_{23}(\tau) \Delta \sigma_{i j}(0)\right\rangle\right], \\
& i, j=2,3 .
\end{aligned}
$$

According to the quantum regression theorem and Eq. (B4), the vectors $\hat{U}^{(i j)}$ satisfy

$$
\frac{d \hat{U}^{(i j)}(\tau)}{d \tau}=B \hat{U}^{(i j)}(\tau), \quad i, j=2,3
$$

By following the same procedure as described in [50], and working in Laplace space we obtain the steady-state fluorescence spectrum

$$
\begin{aligned}
\langle: S(\vec{r}, \omega, \theta):\rangle= & \frac{f(r)^{2}}{4 \pi} \operatorname{Re}\left(\sum _ { k = 1 } ^ { k = 8 } \left\{R_{1 k}(i z)\left[\gamma_{1} \hat{U}_{k}^{(13)}(0)+\gamma_{21} \hat{U}_{k}^{(23)}(0)\right]\right.\right. \\
& \left.+R_{2 k}(i z)\left[\gamma_{2} \hat{U}_{k}^{(13)}(0)+\gamma_{12} \hat{U}_{k}^{(13)}(0)\right]\right\} \\
& \times e^{i 2\left(\theta+\omega_{L} r / c\right)}+\sum_{k=1}\left\{R _ { 1 k } ( i z ) \left[\gamma_{1} \hat{U}_{k}^{(31)}(0)\right.\right. \\
& \left.+\gamma_{21} \hat{U}_{k}^{(32)}(0)\right]+R_{2 k}(i z)\left[\gamma_{2} \hat{U}_{k}^{(32)}(0)\right. \\
& \left.\left.\left.+\gamma_{12} \hat{U}_{k}^{(31)}(0)\right]\right\}\right)
\end{aligned}
$$

where $\hat{U}_{m}^{i j}(0)$ stands for the steady-state $m$ th component of the vector $\hat{U}^{i j}(\tau)$ and $R_{j k}(i z)$ is the $(j, k)$ element of the matrix $R(i z) \equiv\left[(i z \hat{I}-B)^{-1}+(-i z \hat{I}-B)^{-1}\right], \hat{I}$ being the $8 \times 8$ identity matrix and $z \equiv\left(\omega-\omega_{L}\right) / \sqrt{\gamma_{1} \gamma_{2}}$.

\section{APPENDIX C: DECAY RATES OF THE DENSITY MATRIX EQUATIONS IN THE DRESSED-STATE PICTURE}

The $\Gamma$ of Eq. (4.10) are given by

$$
\begin{gathered}
\Gamma_{0}=-\left(\gamma_{1}+\gamma_{2}\right) a_{3 \alpha}^{2}+\left(a_{3 \alpha}^{2}-a_{3 \beta}^{2}\right) \times F, \\
\Gamma_{1}=\left(a_{3 \gamma}^{2}-a_{3 \beta}^{2}\right) \times F, \\
\Gamma_{2}=a_{3 \beta}^{2} \times F, \\
\Gamma_{3}=\left(a_{3 \alpha}^{2}-a_{3 \beta}^{2}\right) \times H,
\end{gathered}
$$

$$
\begin{gathered}
\Gamma_{4}=-\left(\gamma_{1}+\gamma_{2}\right) a_{3 \gamma}^{2}+\left(a_{3 \gamma}^{2}-a_{3 \beta}^{2}\right) \times H, \\
\Gamma_{5}=a_{3 \beta}^{2} \times H,
\end{gathered}
$$

$$
\begin{aligned}
\Gamma_{\alpha \gamma}= & \frac{\gamma_{1}+\gamma_{2}}{2}\left(a_{3 \alpha}^{2}-a_{3 \gamma}^{2}\right)-a_{3 \alpha} a_{3 \gamma}\left(\gamma_{1} a_{1 \alpha} a_{1 \gamma}+\gamma_{2} a_{2 \alpha} a_{2 \gamma}\right) \\
& -\gamma_{12} a_{3 \alpha} a_{3 \gamma}\left(e^{i \delta} a_{1 \alpha} a_{2 \gamma}+e^{-i \delta} a_{1 \gamma} a_{2 \alpha}\right)
\end{aligned}
$$




$$
\begin{aligned}
\Gamma_{\alpha \beta}= & \frac{\gamma_{1}+\gamma_{2}}{2}\left(a_{3 \alpha}^{2}+a_{3 \beta}^{2}\right)-a_{3 \alpha} a_{3 \beta}\left(\gamma_{1} a_{1 \alpha} a_{1 \beta}+\gamma_{2} a_{2 \alpha} a_{2 \beta}\right) \\
& -\gamma_{12} a_{3 \alpha} a_{3 \beta}\left(e^{i \delta} a_{1 \alpha} a_{2 \beta}+e^{-i \delta} a_{1 \beta} a_{2 \alpha}\right), \\
\Gamma_{\beta \gamma}= & \frac{\gamma_{1}+\gamma_{2}}{2}\left(a_{3 \gamma}^{2}+a_{3 \beta}^{2}\right)-a_{3 \gamma} a_{3 \beta}\left(\gamma_{1} a_{1 \gamma} a_{1 \beta}+\gamma_{2} a_{2 \gamma} a_{2 \beta}\right) \\
& -\gamma_{12} a_{3 \gamma} a_{3 \beta}\left(e^{i \delta} a_{1 \beta} a_{2 \gamma}+e^{-i \delta} a_{1 \gamma} a_{2 \beta}\right) .
\end{aligned}
$$

The coefficients $F$ and $H$ in Eq. (C1) are given by

$$
\begin{aligned}
& F=\gamma_{1} a_{1 \alpha}+\gamma_{2} a_{2 \alpha}+2 \gamma_{12} a_{1 \alpha} a_{2 \alpha} \cos (\delta), \\
& H=\gamma_{1} a_{1 \gamma}+\gamma_{2} a_{2 \gamma}+2 \gamma_{12} a_{1 \gamma} a_{2 \gamma} \cos (\delta) .
\end{aligned}
$$

[1] B. R. Mollow, Phys. Rev. 188, 1969 (1969).

[2] C. Cohen Tannoudji, in Frontiers in Laser Spectroscopy, edited by S. Haroche and S. Liberman (North-Holland, New York, 1977).

[3] P. Grangier, G. Roger, A. Aspect, A. Heidmann, and S. Reynaud, Phys. Rev. Lett. 57, 687 (1986).

[4] H. J. Kimble, M. Dagenais, and L. Mandel, Phys. Rev. Lett. 39, 691 (1977).

[5] F. Diedrich and H. Walther, Phys. Rev. Lett. 58, 203 (1987).

[6] J. T. Hoffges, W. Baldauf, T. Eichler, S. R. Helmfrid, and H. Walther, Opt. Commun. 133, 170 (1997).

[7] L. Mandel, Phys. Rev. Lett. 49, 136 (1982).

[8] J. Mod. Opt. 34(6, 7) (1987), special issue on squeezed light, edited by R. Loudon and P. L. Knight; J. Opt. Soc. Am. B 4(10) (1987), special issue on squeezed light, edited by H. J. Kimble and D. F. Walls.

[9] L. Knoll, W. Vogel, and D. G. Welsch, J. Opt. Soc. Am. B 3, 1315 (1986); Phys. Rev. A 42, 503 (1990).

[10] K. W. Vogel and D. G. Welsh, Lectures in Quantum Optics (Akademie-Verlag, Berlin, 1994), Chap. 6.

[11] D. F. Walls and P. Zöller, Phys. Rev. Lett. 47, 709 (1981).

[12] M. J. Collet, D. F. Walls, and P. Zöller, Opt. Commun. 52, 145 (1984).

[13] D. F. Walls and G. J. Milburn, Quantum Optics (Springer Verlag, Berlin, 1994), Chap. 11.

[14] Z. Ficek and S. Swain, J. Opt. Soc. Am. B 14, 258 (1997).

[15] W. Vogel, Phys. Rev. Lett. 67, 2450 (1991).

[16] Z. H. Lu, S. Bali, and J. E. Thomas, Phys. Rev. Lett. 81, 3635 (1998).

[17] H. Z. Zhao, Z. H. Lu, A. M. Bacon, L. J. Wang, and J. E. Thomas, Phys. Rev. A 57, 1427 (1998).

[18] K. Wódkiewicz, P. L. Knight, S. J. Buckle, and S. M. Barnett, Phys. Rev. A 35, 2567 (1987).

[19] M. D. Reid, D. F. Walls, and B. J. Dalton, Phys. Rev. Lett. 55, 1288 (1985).

[20] C. M. Savage and D. F. Walls, Phys. Rev. A 33, 3282 (1985).

[21] W. Vogel and R. Blatt, Phys. Rev. A 45, 3319 (1992).

[22] Z. Ficek, B. J. Dalton, and P. L. Knight, Phys. Rev. A 50, 2594 (1994).

[23] M. O. Scully, Phys. Rep. 219, 191 (1992); O. Kocharovskaya, ibid. 219, 175 (1992); J. Mompart and R. Corbalan, J. Opt. B: Quantum Semiclassical Opt. 27, 175 (2000).

[24] S. E. Harris, J. E. Field, and A. Imamoglu, Phys. Rev. Lett. 64, 1107 (1990).
[25] K. J. Boller, A. Imamoglu, and S. E. Harris, Phys. Rev. Lett. 66, 2593 (1991).

[26] O. Kocharovskaya and Y. I. Khanin, JETP Lett. 48, 580 (1988).

[27] S. E. Harris, Phys. Rev. Lett. 62, 1033 (1989).

[28] M. O. Scully, S.-Y. Zhu, and A. Gavrielides, Phys. Rev. Lett. 62, 2813 (1989).

[29] M. O. Scully, Phys. Rev. Lett. 67, 1855 (1991).

[30] S. E. Harris, J. E. Field, and A. Kasapi, Phys. Rev. A 46, R29 (1992).

[31] M. Fleischhauer, C. H. Keitel, M. O. Scully, Ch Su, B. T. Ulrich, and S.-Y. Zhu, Phys. Rev. A 46, 1468 (1992).

[32] M. O. Scully and M. Fleischhauer, Phys. Rev. Lett. 69, 1360 (1992).

[33] K. Hakuta, L. Marmet, and B. P. Stoicheff, Phys. Rev. Lett. 66, 596 (1991).

[34] S. E. Harris, Phys. Rev. Lett. 72, 52 (1994).

[35] H. Lee, P. Polynkin, M. O. Scully, and S. Y. Zhu, Phys. Rev. A 55, 4454 (1997).

[36] P. Zhou and S. Swain, Phys. Rev. Lett. 77, 3995 (1996).

[37] P. Zhou and S. Swain, Phys. Rev. A 56, 3011 (1997).

[38] S.-Y. Zhu, and M. O. Scully, Phys. Rev. Lett. 76, 388 (1996).

[39] D. A. Cardimona, M. G. Raymer, and C. R. Stroud, Jr., J. Phys. B 15, 55 (1982).

[40] E. Paspalakis and P. L. Knight, Phys. Rev. Lett. 81, 293 (1998).

[41] E. Paspalakis, N. J. Kylstra, and P. L. Knight, Phys. Rev. Lett. 82, 2079 (1999).

[42] U. Akram, Z. Fizec, and S. Swain, J. Mod. Opt. 48, 1059 (2001).

[43] J. Javananien, Europhys. Lett. 17, 407 (1992).

[44] X.-M. Hu and J.-S. Peng, J. Phys. B 33, 921 (2000).

[45] G. S. Agarwal, Quantum Optics, Springer Tracts in Modern Physics Vol. 70 (Springer-Verlag, Berlin, 1974).

[46] S. Menon and G. S. Agarwal, Phys. Rev. A 57, 4014 (1998).

[47] J.-H. Wu and J.-Y. Gao, Phys. Rev. A 65, 063807 (2002).

[48] A. Joshi, W. Yang, and M. Xiao, Phys. Lett. A 315, 203 (2003).

[49] J. Evers, D. Bullock, and C. H. Keitel, Opt. Commun. 209, 173 (2002).

[50] S.-Y. Gao, F.-L. Li, and S.-Y. Zhu, Phys. Rev. A 66, 043806 (2002).

[51] M. A. Antón, F. Carreño, and O. G. Calderón, Opt. Commun. 234, 281 (2004). 
[52] M. O. Scully and M. S. Zubairy, Quantum Optics (Cambridge University Press, London, 1997); J.-S. Peng, and L. GaoXiang, Introduction to Modern Quantum Optics (World Scientific, Singapore, 1998), Chap. 14.

[53] Z. Ficek and P. D. Drummond, Phys. Rev. A 43, 6247 (1991).

[54] H. R. Xia, C. Y. Ye, and S. Y. Zhu, Phys. Rev. Lett. 77, 1032 (1996).

[55] L. Li, X. Wang, J. Yang, G. Lazarov, J. Qi, and A. M. Lyyra, Phys. Rev. Lett. 84, 4016 (2000).
[56] A. K. Patnaik and G. S. Agarwal, Phys. Rev. A 59, 3015 (1999).

[57] G. S. Agarwal, Phys. Rev. Lett. 84, 5500 (2000).

[58] L. M. Narducci, M. O. Scully, G. L. Oppo, P. Ru, and J. R. Tredicce, Phys. Rev. A 42, 1630 (1990).

[59] D. V. Kosachiov, B. G. Matisov, and Y. V. Rodhesttvensky, J. Phys. B 25, 2473 (1992).

[60] E. A. Korsunsky, N. Leinfellner, A. Huss, S. Baluschev, and L. Windholz, Phys. Rev. A 59, 2302 (1999). 